\title{
Contribución de la temperatura fría y el sabor ácido en la intervención fonoaudiológica de la disfagia orofaríngea
}

\section{Contribution of cold Temperature and sour Taste in Speech and Language Intervention of Oropharyngeal Dysphagia}

\section{Exequiel Guevara D. \\ Fonoaudiólogo Universidad Mayor}

\section{Samuel Avendaño S.}

Fonoaudiólogo

I. Municipalidad de Placilla

\author{
Andrea Salazar A. \\ Fonoaudióloga \\ Universidad Santo Tomás
}

\section{María José Alarcón B. Fonoaudióloga}

Corporación Municipal de Villa

Alemana

\section{Macarena Santelices S.}

Fonoaudióloga Centro Comunitario de Salud Familiar Manuel Miranda

Contacto con el autor:

Exequiel Guevara D.

Temuco - Chile

Correo-e: exequiel.gd@gmail.com

Recibido: 01/08/2015 Aceptado: 20/01/2016

\section{RESUMEN}

El fonoaudiólogo es el principal profesional en la rehabilitación no farmacológica y no quirúrgica del usuario con disfagia. Su participación es fundamental tanto para el aminoramiento del riesgo de aspiración o penetración laríngea, como para mejorar o restaurar la función deglutoria. Para este fin, posee opciones terapéuticas directas e indirectas, cuya elección y aplicación dependerá de la patología que curse el usuario, las redes que posea para su recuperación y la motivación intrínseca del mismo. Entre las estrategias de intervención indirecta se encuentra el Tratamiento Sensorio-Motor Oral (OSMT, por sus siglas en inglés), el cual pretende producir una aceleración en el desencadenamiento del proceso deglutorio mediante la ejercitación de los músculos orofaciales en conjunto con diferentes estímulos sensoriales (específicamente la temperatura fría y el sabor ácido). La presente revisión tiene por objetivo dilucidar si la utilización de la temperatura fría y el sabor ácido son útiles como mecanismo de intervención indirecta de la disfagia. Se concluye que las acciones propuestas son efectivas simplemente como mecanismos compensatorios en el proceso deglutorio, puesto que modifican las características del bolo alimenticio e incrementan momentáneamente las sensaciones intraorales.

Palabras clave: ácido, deglución, disfagia, frío, tratamiento sensorio-motor oral.

\section{ABSTRACT}

The speech-language pathologist (SLP) is the main professional in the nonpharmacological and non-surgical rehabilitation of patients with dysphagia. Their role is essential for both reducing the risk of aspiration or laryngeal penetration and improving or restoring the swallowing function. To this end, the SLP has direct and indirect therapeutic options, whose choice and application will depend on the patient's condition, support networks, and their intrinsic motivation. As part of the indirect intervention strategies, the oral sensorymotor treatment (OSMT) aims to exercise the orofacial muscles, and introduce sensory input by the application of cold temperature and sour taste to increase the triggering speed of the swallowing reflex. This review seeks to determine whether the use of cold temperature and sour taste are effective indirect mechanisms for treating patients with dysphagia. It is concluded that the proposed actions in this review are useful simply as compensatory mechanisms in the swallowing process, as they modify the bolus properties and increase, temporarily, the intra-oral sensations.

Keywords: cold temperature, dysphagia, oral sensory-motor treatment, sour taste, swallowing. 


\section{Introducción}

El acto de alimentarse, junto con el objetivo de la nutrición, tiene un carácter cultural muy relevante dentro de la sociedad actual. Por lo tanto, una merma en esta función, además de influir en la ingesta de nutrientes y en la seguridad del proceso alimenticio, puede repercutir negativamente en las interacciones sociales. Es por eso que resulta fundamental la rehabilitación en aquellas personas que presenten cualquier tipo de alteración en el proceso deglutorio.

La disfagia corresponde a la sensación de dificultad en la progresión de la comida desde la boca hacia el estómago, originada por una alteración en alguna de las cuatro etapas del proceso deglutorio (Nazar, Ortega, y Fuentealba, 2009). Se presenta como consecuencia de alteraciones anatómicas en las estructuras involucradas, o más comúnmente por afectaciones en el sistema nervioso central (Hamdy et al., 2003; López, Padín y Bennett, 2004).

Si bien las alteraciones deglutorias son bastante frecuentes en los trastornos neurológicos, puede ocurrir que este trastorno no se relacione directamente con la causa neurológica de base, sino que ocurra de forma secundaria como consecuencia de una posintubación (López et al., 2004) o con posterioridad a la extirpación de un tumor en cabeza o cuello (McCabe et al., 2009; Pauloski, 2008).

La disfagia o dificultad para comer puede tener un efecto devastador en la salud y calidad de vida de las personas, siendo un problema creciente en la población adulta (Lazarus, Clark, Arvedson, Schooling \& Frymark, 2011). El Ministerio de Salud de México (2015) refiere que hasta un tercio de esta población podría presentar algún grado de desnutrición, así como también señala que dos tercios de ellos desarrollarían penetraciones y aspiraciones laríngeas, provocando un elevado riesgo de neumonías por aspiración e infecciones respiratorias. Nazar et al. (2009) manifiestan que la prevalencia de disfagia en la población general oscila entre el $6 \%$ y el $9 \%$, aumentando progresivamente con la edad y llegando a afectar a un $60 \%$ en los adultos mayores que viven en casas de reposo. Además, se señala que las personas de mayor edad reclutarían más áreas corticales que los jóvenes (Humbert et al., 2009). Por su parte, Howden (2004) estima que el $22 \%$ de los adultos sobre los 50 años presentaría algún grado de disfagia.

Una de las patologías neurológicas con más incidencia de disfagia es el accidente cerebrovascular (ACV), en el cual mediante la evaluación con videofluoroscopía (VFC), se indicaría la presencia de disfagia entre un $64 \%$ y un $90 \%$ en aquellas personas post ACV que se encuentren en etapa aguda (Ministerio de Salud de Chile, Minsal, 2013). Además, se destaca que el fonoaudiólogo debe formar parte del equipo multidisciplinario encargado del diagnóstico y rehabilitación de las personas con ACV, tanto en las fases agudas como tardías (Schelp, Cola, Gatto, Silva \& Carvalho, 2004).

En la rehabilitación de la disfagia orofaríngea se han utilizado múltiples técnicas fonoaudiológicas como cambios posturales, modificaciones en la consistencia de la dieta, cambios en los volúmenes de los bolos, así como también estrategias relacionadas con los sabores y las temperaturas para intentar estimular y modular la dinámica de la deglución. 
Guevara, E., Avendaño, S., Salazar, A., Alarcón, M. y Santelices, M.

Este trabajo tiene como objetivo presentar una revisión acerca de la influencia del sabor ácido en conjunto con la temperatura fría en el mecanismo de la deglución. Lo anterior se sustenta en que diversos estudios señalan que el acciomar vinculado de ambas estrategias produciría cambios significativos en los patrones deglutorios, acortando el tiempo de tránsito orofaríngeo.

\section{Metodología}

En primer término, se buscó información de manera sistemática utilizando la base de datos primaria MEDLINE, en donde se seleccionan aquellos artículos que poseían la mayor evidencia científica. Asimismo, el trabajo se complementó con Guías de Práctica Clínica (GPC) de diversas entidades gubernamentales y con artículos aportados por los propios investigadores.

Posteriormente, se efectuó un análisis de la información recopilada, estableciéndose los grados de recomendación a través de consenso entre los investigadores, de acuerdo con los criterios establecidos por el Scottish Intercollegiate Guidelines Network (SIGN) (Tablas 1 y 2) (Manterola y Zavando, 2009; Scottish Intercollegiate Guidelines Network, 2014).

Tabla 1

Niveles de evidencia científica

\begin{tabular}{cl}
\hline $1++$ & $\begin{array}{l}\text { Metaanálisis de alta calidad, revisiones sistemáticas de ensayos clínicos o ensayos clínicos de alta calidad con } \\
\text { muy poco riesgo de sesgos. }\end{array}$ \\
\hline $\mathbf{1 +}$ & $\begin{array}{l}\text { Metaanálisis bien realizados, revisiones sistemáticas de ensayos clínicos o ensayos clínicos bien realizados con } \\
\text { poco riesgo de sesgos. }\end{array}$ \\
\hline $\mathbf{* 1 -}$ & Metaanálisis, revisiones sistemáticas de ensayos clínicos o ensayos clínicos con alto riesgo de sesgos. \\
\hline $\mathbf{2 + +}$ & $\begin{array}{l}\text { Revisiones sistemáticas de alta calidad de estudios de cohortes o de casos y controles. Estudios de cohortes o } \\
\text { de casos y controles con riesgo muy bajo de sesgo y con alta probabilidad de establecer una relación causal. }\end{array}$ \\
\hline $\mathbf{2 +}$ & $\begin{array}{l}\text { Estudios de cohortes o de casos y controles bien realizados con bajo riesgo de sesgo y con una moderada } \\
\text { probabilidad de establecer una relación causal. }\end{array}$ \\
\hline $\mathbf{2 -}$ & $\begin{array}{l}\text { Estudios de cohortes o de casos y controles con alto riesgo de sesgo y riesgo significativo de que la relación no } \\
\text { sea causal. }\end{array}$ \\
\hline $\mathbf{3}$ & Estudios no analíticos, como informes de casos y series de casos. \\
\hline $\mathbf{4}$ & Opinión de expertos. \\
\hline
\end{tabular}

Tabla 2

Grados de recomendación

\begin{tabular}{cl}
\hline A & $\begin{array}{l}\text { Al menos un metaanálisis, revisión sistemática o ensayo clínico clasificado como 1++ y directamente aplicable } \\
\text { a la población diana de la guía; o un volumen de evidencia científica compuesto por estudios clasificados } \\
\text { como 1+ y con gran consistencia entre ellos. }\end{array}$ \\
\hline B & $\begin{array}{l}\text { Un volumen de evidencia compuesto por estudios clasificados como 2++, directamente aplicable a la } \\
\text { población diana de la guía y que demuestran gran consistencia entre ellos; o evidencia científica extrapolada } \\
\text { desde estudios clasificados como 1++ o 1+. }\end{array}$ \\
C & $\begin{array}{l}\text { Un volumen de evidencia científica compuesto por estudios clasificados como 2+ directamente aplicables a la } \\
\text { población diana de la guía y que demuestran gran consistencia entre ellos; o evidencia científica extrapolada }\end{array}$ \\
\hline D & desde estudios clasificados como 2++. \\
\hline * Los estudios clasificados como 1-y 2- no deben utilizarse en la elaboración de recomendaciones por el alto nivel de sesgos que poseen.
\end{tabular}




\section{Etapas de la deglución}

Nazar et al. (2009) expone que la deglución se divide en cuatro etapas sucesivas: fase preparatoria oral, fase oral, fase faríngea y fase esofágica.

a) Fase preparatoria oral voluntaria. Presenta una duración variable y se inicia con la introducción del alimento a la boca. Concluye en el proceso de masticación y con la preparación del bolo alimenticio mezclado con la saliva.

b) Fase oral. Esta etapa dura un segundo y es también de control voluntario. Se manifiesta con la propulsión del bolo alimenticio hacia posterior, siendo empujado por un movimiento de elevación del ápex lingual y de propulsión anteroposterior, asociado a un retroceso de la raíz de la lengua.

c) Fase faríngea. Etapa conocida como automáticorefleja. Comienza con el mecanismo complejo e indispensable que constituye el reflejo de deglución, provocando una serie de modificaciones perfectamente coordinadas para guiar el bolo alimenticio desde la boca hacia el esófago superior. Las acciones que ocurren son: elevación del velo del paladar (cierre de rinofaringe), apertura del esfínter esofágico superior (EES), cierre de la glotis y ascenso laríngeo, propulsión lingual y contracción faríngea.

d) Fase esofágica. Esta etapa es esencialmente refleja y escapa de la voluntad. Dura entre 6 y 8 segundos y comienza con el paso del alimento al esófago. Las ondas peristálticas y la apertura del Esfínter Esofágico Inferior (EEI) permiten que el bolo alcance el estómago. Con esta fase se da comienzo al largo proceso de la digestión.

\section{Neuroanatomía de la deglución}

La cavidad oral, la faringe y la laringe constituyen una de las zonas más ricas y diversas en receptores sensoriales de todo el cuerpo, lo que se evidencia en la densa e intrincada inervación que reciben (Mu \& Sanders, 2000). Estas regiones poseen un amplio rango de modalidades, incluyendo la discriminación de dos puntos, detección vibro-táctil, sensibilidad somestésica, propiocepción, nocicepción, sensibilidad química y sensibilidad térmica (Dodds et al., 1988; Miller, 2002).

La estimulación mecánica y química de la mucosa faríngea y laríngea ayudan a desencadenar el reflejo de la deglución (Kajii et al., 2002). Pommerenke (1928) citado por Kajii et al. (2002), encontró que los pilares de las fauces son las regiones más sensitivas a la estimulación de presión suave en humanos. También señaló que la pared posterior de la faringe es altamente sensible a la presión, pero requiere de una intensidad mucho mayor que la necesaria para que los pilares respondan. Storey (1968), por su parte, reveló que la estimulación con agua es más efectiva que la estimulación mecánica en la mucosa laríngea.

Los Pilares Anteriores de las Fauces (AFP, por sus siglas en inglés) son bilaterales y se encuentran en la cavidad oral a cada lado del velo, formando parte del paladar blando. Son inervados por la rama maxilar del nervio trigémino y el nervio glosofaríngeo. Estudios clínicos han demostrado que la estimulación táctil de los AFP aumenta la velocidad para deglutir, facilitando la deglución por varios minutos (Teismann et al., 2009).

Las regiones efectivas para la evocación del reflejo deglutorio son inervadas por el Nervio 
Glosofaríngeo (GPN, por sus siglas en inglés) y el Nervio Laríngeo Superior (SLN, por sus siglas en inglés). El SLN es el nervio más importante para el inicio del reflejo de la deglución de la laringe (Kajii et al., 2002). Por su parte, Kitagawa et al. (2009), evidenciaron que la efectividad de la rama faríngea del GPN (GPNph) en la evocación de la deglución en respuesta a la estimulación eléctrica es comparable con la del SLN, y determinaron que el GPNph cumple un rol mayor en el desencadenamiento del reflejo deglución de la faringe.

En la deglución orofaríngea, los estímulos sensitivos son transmitidos de manera aferente por medio de los pares craneanos V, VII, IX y X hacia el núcleo del tracto solitario y núcleo ambiguo del tronco encefálico. En este lugar se coordinará una respuesta motora eferente que se dirigirá por los pares V, VII, IX, X y XII, controlando la actividad muscular oral y faríngea. Se señala que las fases preparatoria oral y oral, al ser voluntarias, se encontrarían controladas por la corteza frontal (Nazar et al., 2009).

Dziewas et al. (2003), en tanto, destacan y sugieren que en la deglución voluntaria existe una fuerte lateralización hacia el hemisferio izquierdo dominante, involucrando múltiples áreas cerebrales, tales como el área sensorio-motora primaria, el área de integración somatosensorial, la ínsula, el opérculo frontal y el giro temporal superior. Complementando lo anterior, Dziewas et al. (2003), mencionan que el giro del cíngulo, el cuerpo calloso, el tálamo, los núcleos basales y el cerebelo también contribuyen a la organización central de la deglución.

\section{Intervención indirecta de la disfagia}

Lazarus et al. (2011), refiere que por años los fonoaudiólogos han implementado un tratamiento integral a los adultos con disfagia, ofreciendo un número de intervenciones para mejorar la función deglutoria. Nazar et al. (2009) señalan que la rehabilitación fonoaudiológica de la deglución se divide en un tratamiento indirecto y otro directo. El tratamiento indirecto es realizado sin alimentos y consiste en ejercicios para mejorar la motricidad oral, faríngea y laríngea. Sumado a lo anterior, se efectúa deglución de saliva y estimulación térmica y con sabores. Además, se les enseña a las personas maniobras para prevenir la aspiración, como la doble deglución. En cambio, en el tratamiento directo sí se utilizan alimentos e incluyen ejercicios motores orales, técnicas posturales compensatorias, maniobras de deglución y modificación de la dieta.

Lazarus et al. (2011) señalan, además, que el Tratamiento Sensorio-Motor Oral (OSMT, por sus siglas en inglés), comúnmente denominado como "ejercicios motores orales", ha sido aceptado ampliamente en la práctica de los fonoaudiólogos desde hace mucho tiempo. Estos ejercicios tienen como objetivo trabajar sobre los labios, lengua, cara, paladar o cuello/laringe y están diseñados para aumentar la movilidad, fuerza y el control de la deglución.

Otros tipos de OSMT han introducido estímulos sensoriales a la orofaringe, incluyendo la mecánica, térmica (por ejemplo frío y calor) y la gustativa (por ejemplo dulce, salado, amargo y ácido). Estos tratamientos sensoriales han sido diseñados tanto para mejorar el desencadenamiento y el tiempo de respuesta motora faríngea, como para aumentar los 
aspectos biomecánicos y la duración de la deglución (Lazarus et al., 2011).

Dada la compleja naturaleza de la disfagia, a menudo las OSMTs son combinados, por ejemplo, utilizando estimulación térmica y ejercicios de fuerza lingual, o son aplicados a la par con otros tratamientos deglutorios, por ejemplo las posturas/maniobras compensatorias y las modificaciones en la dieta. Sin embargo, a pesar de su uso generalizado, se conoce muy poco acerca de sus efectos en la deglución de la población adulta (Lazarus et al., 2011) o los beneficios que traería a la fisiología deglutoria en personas con o sin patología (Hamdy et al., 2003).

Una de las intervenciones que también utiliza la estimulación a través de sensaciones táctiles y térmicas es la Tactile Thermal Oral Stimulation (TTOS) (Teismann et al., 2009), que se realiza acariciando los pilares anteriores de las fauces del usuario con un bastoncillo helado. La superficie del bastoncillo se debe encontrar entre los $-1^{\circ}$ y $\operatorname{los} 3^{\circ} \mathrm{C}$. Ambos pilares son acariciados en series, comenzando con el lado del sujeto que se encuentre alterado. Se frota suavemente desde arriba (medial) hasta abajo (lateral) y no se debe tocar la lengua con el bastón helado. Después de trabajar en ambos lados, se solicita al usuario a deglutir para eliminar el agua derretida. Este ejercicio se realiza cinco veces con una pausa de dos minutos entre series.

\section{¿Contribuye el sabor ácido y la temperatura fría a la intervención fonoaudiológica?}

Si bien en la intervención de la disfagia se utilizan diversas técnicas de rehabilitación y/o compensación, en las que se incluyen las modificaciones gustativas y de la temperatura, estas aún no han demostrado su efectividad para ser implementadas en todos los usuarios portadores de esta condición.

Diversos estudios han intentado dilucidar la efectividad de ciertos procedimientos que realizan los profesionales fonoaudiólogos para la intervención de sus usuarios. Sin embargo, muchas veces se ejecutan ejercicios que no cuentan con un respaldo científico y se guían netamente por la práctica clínica cotidiana (Tan, Liu, Li, Liu \& Chen, 2013).

Por otra parte, existe escasez de evidencia para la terapia de la disfagia, por tanto se hace difícil determinar la mejor opción para efectuar el tratamiento (Archer, Wellwood, Smith \& Newham, 2013).

Es importante recordar que la disfagia puede ocurrir secundariamente como resultado de algún deterioro neurológico, de cambios estructurales y/o enfermedades progresivas, teniendo el potencial de impactar negativamente en la nutrición, hidratación y calidad de vida de las personas (Molfenter \& Steele, 2012). Además se debe mencionar que pueden originarla múltiples patologías, las que no discriminan edad de presentación ni género.

Morgan, Dodrill y Ward (2012) concluyen que existe insuficiente evidencia de calidad en la terapia motora-oral para los niños que presenten afectación neurológica y destacan la urgente necesidad de realizar estudios de ensayos clínicos aleatorizados para evaluar la efectividad de las intervenciones en la disfagia orofaríngea. 
Por tanto, aunque se menciona la utilización conjunta de la estimulación táctil y térmica sobre los pilares anteriores como un mecanismo para acelerar la respuesta faríngea deglutoria en personas neurológicamente dañadas con un retraso en el inicio deglutorio, la eficacia de esta estrategia y su éxito a largo plazo aún no se ha demostrado (Sciortino, Liss, Case, Gerritsen \& Katz, 2003).

Se señala, entonces, que más que la ejecución de intervención indirecta aplicando temperatura fría y sabor ácido, estas modificaciones de deben incorporar de manera compensatoria y directamente en el bolo alimenticio, con el fin de aumentar $y$ estimular las diferentes sensaciones intraorales (Calixto-Lima, Martins de Andrade, Gomes, Geller \& Siqueira-Batista, 2012; College of Audiologists and Speech-Language Pathologists of Ontario, 2007; Intercollegiate Stroke Working Party, 2012; The Speech Pathology Association of Australia Limited, 2012).

Cabe destacar que las técnicas compensatorias se refieren a aquellas que, cuando se aplican, tienen un efecto inmediato, pero normalmente transitorio sobre la eficacia o la seguridad de la deglución. Como su nombre lo indica, estas técnicas se aplican directamente en el bolo alimenticio, siendo utilizadas para compensar (no para remediar), anormalidades de la deglución (The Speech Pathology Association of Australia Limited, 2012).

La mayoría de los estudios señala una carencia en la evidencia científica en los procedimientos utilizados para la intervención de la disfagia. Concuerdan también en que la estimulación de la temperatura fría y del sabor ácido como estrategias rehabilitadoras adolecen de un respaldo científico, por tanto se deberían eliminar del abanico de opciones terapéuticas, comenzando a abordar solo aquellos procedimientos que poseen un mayor sustento.

\section{Recomendaciones}

Existe insuficiente información para recomendar la estimulación física (térmica, táctil) para la intervención de la disfagia en personas secueladas de ACV (Geeganage, Beavan, Eliender \& Bath, 2012) (Recomendación B).

Restricciones en la textura de la dieta y las modificaciones de los alimentos son las formas más comunes de manejo compensatorio en personas con disfagia (College of Audiologists and SpeechLanguage Pathologists of Ontario, 2007) (Recomendación B).

Dentro de las estrategias compensatorias se proponen las técnicas comportamentales o de estimulación para desencadenar de manera oportuna el proceso deglutorio (por ejemplo, la estimulación termo-táctil, el sabor ácido o bolos carbonatados (College of Audiologists and SpeechLanguage Pathologists of Ontario, 2007) (Recomendación B).

Como mecanismo compensatorio, se propone ajustar la sensibilidad de los líquidos y las comidas, mediante bolos alimenticios fríos, ácidos o carbonatados (The Speech Pathology Association of Australia Limited, 2012) (Recomendación D).

Las intervenciones térmica y química proveen un tratamiento paliativo en las personas con disfagia posterior a un cáncer esofágico, sin embargo desencadenan un aumento en las reintervenciones y en la presencia de efectos adversos (Dai et al., 2014) (Recomendación B). 
En el caso de las personas en tratamiento de quimioterapia que cursen con disfagia por complicaciones gastrointestinales se debe aumentar la frecuencia de alimentación, servir el alimento en pequeñas cantidades y evitar las temperaturas extremas (Calixto-Lima et al., 2012) (Recomendación C).

Se sugieren, así mismo, modificaciones sensoriales de los alimentos para personas con un $A C V$, ya sea alterando el sabor y la temperatura, o bien, por medio de líquidos carbonatados (Intercollegiate Stroke Working Party, 2012) (Recomendación B).

Estímulos altamente ácidos podrían contribuir a aumentar el riesgo de neumonías por aspiración en que aquellas personas que se encuentren aspirando (College of Audiologists and Speech-Language Pathologists of Ontario, 2007) (Recomendación B).

Por último, el mejor ejercicio para mejorar la deglución es la deglución (The Speech Pathology Association of Australia Limited, 2012) (Recomendación B).

\section{Comentarios finales}

En reiteradas ocasiones los profesionales
fonoaudiólogos ejecutan intervenciones sin
cuestionar la real efectividad de esa acción
terapéutica, o si las mismas fueron
diseñadas para esa condición de base o rango
etario.
Se evidencia que
intervención indirecta y reactivadora, la utilización
de la temperatura fría y el sabor ácido no poseen
suficiente respaldo científico que las avale como
tales, por tanto, no debiesen ser recomendadas para

su inclusión en la intervención de las personas con disfagia.

Sin embargo, se podrían sugerir variaciones en la temperatura y los sabores de manera compensatoria para modificar el bolo alimenticio, estimulando y aumentando momentáneamente las sensaciones intraorales.

Al finalizar, cabe señalar que la intervención en la disfagia aún es un tema pendiente, en donde son necesarios mayores estudios que permitan esclarecer, con evidencia científica de alta calidad, los mejores procedimientos para ser utilizados en la rehabilitación del proceso deglutorio. 


\section{Referencias}

Archer, S., Wellwood, I., Smith, C., \& Newham D. (2013). Dysphagia therapy in stroke: A survey of speech and language therapists. International Journal of Language \& Communication Disorders, 48(3), 283-296. http://dx.doi.org/10.1111/1460-6984.12006

Calixto-Lima, L., Martins de Andrade, E., Gomes, P., Geller, M., \& Siqueira-Batista, R. (2012). Dietetic management in gastrointestinal complications from antimalignant chemotherapy. Nutrición Hospitalaria, 27(1), 65-75. Recuperado de http://www.redalyc.org/pdf/3092/309226784008.pdf

College of Audiologists and Speech-Language Pathologists of Ontario. (2007). Practice Standards and Guidelines for Dysphagia Intervention by Speech-Language Pathologists. Toronto. Recuperado de http://www.caslpo.com/sites/default/uploads/files/PSG E N Dysphagia.pdf

Dai, Y., Li, C., Xie, Y., Liu, X., Zhang, J., Zhou, J., Pan, X., \& Yang, S. (2014). Interventions for dysphagia in oesophageal cancer. Cochrane Database of Systematic Reviews, 10, 1-3. http://dx.doi.org/10.1002/14651858.cd005048.pub4

Dodds, W., Man, K., Cook, I., Kahrilas, P., Stewart, E., \& Kern, M. (1988). Influence of bolus volume on swallowinduced hyoid movement in normal subjects. American Journal of Roentgenology, 150(6), 1307-1309. http://dx.doi.org/10.2214/ajr.150.6.1307

Dziewas, R., Sörös, P., Ishii, R., Chau, W., Henningsen, H., Ringelstein, E. B., Knecht, S., \& Pantev, C. (2003). Neuroimaging evidence for cortical involvement in the preparation and in the act of swallowing. Neurolmage, 20(1), 135-144. http://dx.doi.org/10.1016/s10538119(03)00285-4

Geeganage, C., Beavan, J., Ellender, S., \& Bath P. M. W. (2012). Interventions for dysphagia and nutritional support in acute and subacute stroke. Cochrane Database of $\begin{array}{llll}\text { Systematic } & \text { Reviews, } & 10, & 1-97 .\end{array}$ http://dx.doi.org/10.1002/14651858.cd000323.pub2

Hamdy, S., Jilani, S., Price, V., Parker, C., Hall, N., \& Power, M. (2003). Modulation of human swallowing behaviour by thermal and chemical stimulation in health and after brain injury. Neurogastroenterology and Motility, 15(1), 69-77. http://dx.doi.org/10.1046/j.1365-2982.2003.00390.x
Howden, C. (2004). Management of acid-related disorders in patients with dysphagia. The American Journal of Medicine, 117(5), 44-48. http://dx.doi.org/10.1016/j.amjmed.2004.07.017

Humbert, I. A., Fitzgerald, M. E., McLaren, D. G., Johnson, S., Porcaro, E., Kosmatka, K., Hind, J., \& Robbins, J. (2009). Neurophysiology of swallowing: Effects of age and bolus type. Neuroimage, 44(3), 982-991. http://dx.doi.org/10.1016/j.neuroimage.2008.10.012

Intercollegiate Stroke Working Party. (2012). National clinical guideline for stroke ( $4^{\text {th }}$ ed.). London: Royal College of Physicians.

Kajii, Y., Shingai, T., Kitagawa, J., Takahashi, Y., Taguchi, Y., Noda, T., \& Yamada, Y. (2002). Sour taste stimulation facilitates reflex swallowing from the pharynx and larynx in the rat. Physiology \& Behavior, 77(2-3), 321-325. http://dx.doi.org/10.1016/s0031-9384(02)00854-5

Kitagawa, J., Nakagawa, K., Hasegawa, M., Iwakami, T., Shingai, T., Yamada, Y., \& Iwata, K. (2009). Facilitation of reflex swallowing from the pharynx and larynx. Journal of Oral Science, 51(2), 167-171. http://dx.doi.org/10.2334/josnusd.51.167

Lazarus, C., Clark, H., Arvedson, J., Schooling, T., \& Frymark, T. (2011). Evidence-based systematic review: Effects of oral sensory-motor treatment on swallowing in adults. ASHA's National Center for Evidence-Based Practice in Communication Disorders, 1-42.

López, M., Padín, M. C., y Bennett, E. (2004). Presencia de la disfagia en los trastornos neurológicos de la comunicación. Revista Mexicana de Neurociencia, 5(5), 455-459. Recuperado de http://revmexneuroci.com/wpcontent/uploads/2014/07/Nm0045-09.pdf

Manterola, C. y Zavando, D. (2009). Cómo interpretar los "Niveles de Evidencia" en los diferentes escenarios clínicos. Revista Chilena de Cirugía, 6, 582-595. http://dx.doi.org/10.4067/s0718-40262009000600017

McCabe, D., Ashford, J., Wheeler-Hegland, K., Frymark, T., Mullen, R., Musson, N., Smith, C., \& Schooling, T. (2009). Evidence-based systematic review: Oropharyngeal dysphagia behavioral treatments. Part IV-Impact of dysphagia treatment on individual's postcancer treatments. Journal of Rehabilitation Research \& Development, 46(2), 205-214. http://dx.doi.org/10.1682/jrrd.2008.08.0092 
Miller, A. (2002). Oral and pharyngeal reflexes in the mammalian nervous system: Their diverse range in complexity and the pivotal role of the tongue. Critical Reviews in Oral Biology \& Medicine, 13(5), 409-425. http://dx.doi.org/10.1177/154411130201300505

Ministerio de Salud de Chile, Minsal. (2013). Guía clínica accidente vascular isquémico en personas de 15 años y más. Recuperado de http://www.supersalud.gob.cl/difusion/572/articles645 recurso $1 . p d f$

Molfenter, S. \& Steele, C. (2012). Temporal variability in the deglutition literature. Dysphagia, 27(2), 162-177. http://dx.doi.org/10.1007/s00455-012-9397-x

Morgan, A., Dodrill, P., \& Ward, E. (2012). Interventions for oropharyngeal dysphagia in children with neurological impairment. Cochrane Database of Systematic Reviews, 10, 1-34. http://dx.doi.org/10.1002/14651858.cd009456.pub2

$\mathrm{Mu}$, L. \& Sanders, I. (2000). Sensory nerve supply of the human oro- and laryngopharynx: A preliminary study. The Anatomical Record, 258(4), 406-420. http://dx.doi.org/10.1002/(sici)10970185(20000401)258:4\%3C406::aid-ar9\%3E3.0.co;2-5

Nazar, G., Ortega, A., y Fuentealba, I. (2009). Evaluación y manejo integral de la disfagia orofaríngea. Revista Médica Clínica Las Condes, 20(4), 449-457. Recuperado de http://www.clc.cl/Dev CLC/media/Imagenes/PDF\%20revis ta\%20m\%C3\%A9dica/2009/4\%20julio/449 DISFAGIA ORO FARINGEA-9.pdf

Pauloski, B. R. (2008). Rehabilitation of dysphagia following head and neck cancer. Physical Medicine and Rehabilitation Clinics of North America, 19(4), 889-928. http://dx.doi.org/10.1016/j.pmr.2008.05.010

Pommerenke, W. (1928). A study of the sensory areas eliciting the swallowing reflex. American Journal of Physiology, 84, 36-41.

Sciortino, K., Liss, J., Case, J., Gerritsen, K., \& Katz, R. (2003). Effects of mechanical, cold, gustatory, and combined stimulation to the human anterior faucial pillars. Dysphagia, 18(1), 16-26. http://dx.doi.org/10.1007/s00455-002-0076-1
Schelp, A., Cola, P., Gatto, A., Silva, R., \& de Carvalho, L. (2004). Incidência de disfagia orofaríngea após acidente vascular encefálico em hospital público de referência. Revista Arquivos de Neuro-psiquiatría, 62(2-B), 503-506. http://dx.doi.org/10.1590/s0004-282x2004000300023

Scottish Intercollegiate Guidelines Network. (2014). SIGN 50, A guideline developer's handbook. Recuperado de http://www.sign.ac.uk/pdf/sign50.pdf

Storey, A. (1968). Laryngeal Initiation of Swallowing. Experimental Neurology, 20(3), 359-365. http://dx.doi.org/10.1016/0014-4886(68)90079-4

Tan, C., Liu, Y., Li, W., Liu, J., \& Chen, L. (2013). Transcutaneous neuromuscular electrical stimulation can improve swallowing function in patients with dysphagia caused by non-stroke diseases: A meta-analysis. Journal of Oral Rehabilitation, 40(6), 472-480. http://dx.doi.org/10.1111/joor.12057

Teismann, I. K., Steinsträter, O., Warnecke, T., Suntrup, S., Ringelstein, E. B., Pantev, C., \& Dziewas, R. (2009). Tactile thermal oral stimulation increases the cortical representation of swallowing. BMC Neuroscience, 10(1), 71. http://dx.doi.org/10.1186/1471-2202-10-71

The Speech Pathology Association of Australia Limited. (2012). Dysphagia Clinical Guideline. Recuperado de http://www.speechpathologyaustralia.org.au/library/Clinic alGuidelines/FINAL15062012DysphagiaClinicalGuidelines.p df. 